\title{
Correction to: Interactive Granular Computing
}

Andrzej Skowron and Andrzej Jankowski

\section{Correction to: \\ Chapter "Interactive Granular Computing" \\ in: D. Ciucci et al. (Eds.): Rough Sets \\ and Knowledge Technology, LNAI 9436, https://doi.org/10.1007/978-3-319-25754-9_5}

The acknowledgement section of this paper originally referred to grant DEC-2013/09/B/ST6/01568. The reference to this grant has been removed from the acknowledgement section at the request of one of the authors. 